\title{
Práctica de materias coordinadas en aprendizaje y servicio: la destreza profesional al servicio de la sociedad próxima
}

\author{
Martínez Costa, Sandra; ${ }^{2}$ Nozal Cantarero, Teresa ${ }^{1}$ \\ ${ }^{1}$ Universidade da Coruña, Facultade de Ciencias da Comunicación, \\ Código ORCID0000-0002-2652-5898 \\ ${ }^{2}$ Universidade da Coruña, Facultade de Ciencias da Comunicación
}

\section{RESUMEN}

Las asignaturas Publicidad Audiovisual y Ficción Audiovisual, ambas impartidas en el primer cuatrimestre de segundo curso del grado en Comunicación Audiovisual de la Universidade da Coruña, se coordinaron en el curso 2018-19 para ofrecer a los alumnos una práctica conjunta en servicio de la sociedad de la ciudad de A Coruña. La esencia del ejercicio consistía en que los estudiantes pusiesen las destrezas profesionales que adquirían en ambas materias al servicio de los centros de acogida de animales de la ciudad y sus alrededores. De esta forma se fomentaba la "autoconciencia" del trabajo profesional como un bien para el entorno social, además de como un medio de subsistencia personal y, a la vez, se formaba en valores a los estudiantes como metodología de innovación docente.

PALABRAS CLAVE: Aprendizaje-Servicio, comunicación audiovisual, Publicidad Audiovisual, Ficción Audiovisual, aprendizaje basado en proyectos. 


\section{CITA RECOMENDADA:}

Martínez Costa, Sandra; Nozal Cantarero, Teresa (2020): Práctica de materias coordinadas en aprendizaje y servicio: la destreza profesional al servicio de la sociedad próxima. En De la Torre Fernández, E. (ed.) (2020). Contextos universitarios transformadores: Boas prácticas no marco dos GID. IV Xornadas de Innovación Docente. Cufie. Universidade da Coruña. A Coruña (págs. 345-358). DOI capítulo: https://doi.org/10.17979/spudc.9788497497756.345 DOl libro: https://doi.org/10.17979/spudc.9788497497756

\section{ABSTRACT}

Audio-visual advertising and audio-visual fiction subjects are both taught in the first four-month term of the Audio-visual Communication Degree of the University of A Corunha. Both Professors of the subjects coordinated in the 2018-19 academic year a joint practice for the students in order to offer a society service to the A Coruña citizens. The essence of that practice was to use the professional skills the students acquired in both subjects for the service of the animal reception centers in the city and its surroundings. In this way, the "self-awareness" of their professional work was promoted as a good for the social environment, as well as as a means of personal subsistence and, at the same time, students were trained in values as aninnovation teaching methodology.

KEY WORDS: service-learning, audio-visual communication, advertising, fiction, project based learning. 


\section{INTRODUCCIÓN}

La experiencia de innovación docente aquí presentada se basa en el aprendizaje a través del desarrollo profesional de destrezas aplicadas al servicio de la sociedad. Dicho de otra manera, se centra en el denominado Aprendizaje-Servicio (ApS), metodología docente que se fundamenta en el trabajo activo del alumno y en su participación colaborativa con el entorno cercano. La finalidad de este tipo de procedimientos es la de fomentar el civismo y la responsabilidad social del estudiante, fortaleciendo su espíritu crítico (Villa, A \& Villa, 0., 2007), sus habilidades y destrezas profesionales, y mejorando al mismo tiempo la calidad de vida de las personas de su realidad social más próxima (Puig, J.M. et al., 2009).

Esta metodología basa el aprendizaje del estudiante en la detección de necesidades reales del entorno más próximo, vinculando la formación del alumno con la solución a esos problemas y necesidades a partir de la participación activa en proyectos en los que puedan desarrollar las competencias adquiridas en su formación académica. Por lo tanto, el Aprendizaje-Servicio suele basarse de manera habitual en el aprendizaje experiencial o project based learning (PBL), que promueve en el estudiante una formación activa, crítica y creativa a través de la realización de proyectos (Marti, J. A.; Heydrich, M.; Rojas, M.; Hernández, A., 2010). El aprendizaje experiencial es por lo tanto imprescindible para que se dé la necesaria asociación entre la formación académica y la formación en valores que demanda el ApS. Esta unión es primordial para que el alumno identifique el conocimiento adquirido en el aula con el compromiso social que tendrá en el desarrollo profesional de su trabajo.

Para que una actividad pueda ser considerada de Aprendizaje-Servicio, esta debe servir para ayudar a mejorar un problema o necesidad detectado, partir como iniciativa de las instituciones académicas y, a su vez, formar parte del plan curricular del estudiante en su formación profesional (Sandrea, L.; Reyes, L., 2010)

Este planteamiento se enmarca también en el modelo universitario de aprendizaje basado en competencias, es decir, en la adquisición de habilidades prácticas y aptitudes trasversales al 
conjunto del estudiantado, y que sirven para su formación como personas y ciudadanos, al margen de su habilitación profesional (Francisco, A.; Moliner, L. 2010)

\section{CONTEXTUALIZACIÓN DE LA EXPERIENCIA DOCENTE}

Las materias de Ficción Audiovisual y Publicidad Audiovisual forman parte de las materias del segundo curso, primer cuatrimestre, del grado en Comunicación Audiovisual de la Universidade da Coruña. Ambas están planteadas para que el estudiante conozca las claves de los códigos de ficción y publicitario, respectivamente. Constan de 6 créditos ECTS y se imparten en el mismo cuatrimestre que otras de formación sobre el código informativo (Xornalismo Audiovisual), estudios sobre las audiencias (Estudos das Audiencias) y la materia de Infografía 3D. Para alcanzar la calificación de apto en cada una de las materias, el estudiante deberá realizar una serie de trabajos prácticos que indiquen que han alcanzado las destrezas suficientes para desenvolverse con soltura en el código publicitario y en el proceso de creación de productos de ficción, así como sendos exámenes sobre el temario visto en el aula. Parte de ese contenido práctico de cada una de las dos asignaturas fue la realización de un proyecto transversal a ambas, en el que los estudiantes actuasen con diferentes roles profesionales y elaborasen una campaña en redes sociales para fomentar la adopción de mascotas en la ciudad de A Coruña y su entorno cercano.

La finalidad era la de conseguir que el estudiante desarrollase destrezas profesionales a través del Aprendizaje-Servicio, del aprendizaje basado en proyectos y del aprendizaje colaborativo, ya que además todos los trabajos debían hacerse en equipo.

El aprendizaje en valores se hizo también fundamental, al tratarse de un proyecto real de servicio social. La colaboración entre las dos materias tuvo la finalidad de que los estudiantes pudiesen elaborar una campaña publicitaria completa, lo cual es un trabajo muy laborioso para un solo cuatrimestre. Por ello, los alumnos realizaban en la materia de ficción los anuncios que luego se utilizarían como anuncios de la campaña que cada uno de los grupos trabajaba desde la materia de Publicidad Audiovisual. 
La práctica consistió en un ejercicio de rol profesional por el cual:

Los alumnos de publicidad actuaban como agencias contratadas para crear en redes sociales una campaña de fomento de la adopción de animales en A Coruña. Las agencias contaban con un supuesto cliente: el propio Ayuntamiento. Para cumplir con el encargo debían definir las ideas para un anuncio, más un corto de ficción que encargaban a una productora audiovisual, y en el que debía aparecer la adopción como eje narrativo y la protectora o perrera como imagen concreta.

- Los alumnos de ficción actuaban como productoras que debían crear sendos productos audiovisuales satisfaciendo las condiciones y necesidades publicitarias de la agencia que les contrataba.

Todos los alumnos actuaban con ambos roles (agencia publicitaria y productora) pero nunca trabajando para un mismo proyecto/ejercicio a la vez, ni siquiera con relación de reciprocidad entre agencia y productora. Las profesoras tutorizaron todo el proceso, hicieron clases conjuntas y elaboraron un calendario con hitos y entregas en las distintas fases del proyecto, adaptándolas a las etapas habituales de un trabajo profesional. A la vez supervisaron la ejecución en redes sociales y la relación establecida entre las protectoras, los estudiantes, y los ciudadanos que intervenían como usuarios y espectadores de la campaña.

Para la elaboración del trabajo las fases fueron las siguientes:

1. Las docentes de las dos materias establecieron el tema, los calendarios, los hitos y entregas, las reuniones entre los distintos equipos de trabajo y las clases conjuntas en las que se hacían presentaciones públicas, así como la distribución de la evaluación.

2. Se establecieron los grupos de trabajo en los turnos de prácticas elaborados por el centro. Las clases prácticas eran de cuatro horas a la semana por cada uno de los grupos.

3. Los estudiantes de la materia de publicidad eligieron la protectora a la que representaban, y plantearon una idea fuerza, un público objetivo, un slogan y una idea 
de guion publicitario. Presentaron su briefing formal a los grupos de ficción y a las profesoras un mes y medio después de iniciado el curso. En esta fecha se incorporaron los estudiantes de ficción a la práctica común.

4. Los estudiantes de la materia de ficción desarrollaron los guiones, los storyboards y el concepto estético-visual que debían presentar en un pitching a los grupos de publicidad. Estos a su vez aprobaban o corregían las ideas propuestas.

5. Los grupos de ficción llevaban a cabo el casting, el diseño de producción, la búsqueda de localizaciones y la realización de los cortos y anuncios publicitarios.

6. Paralelamente, los grupos de publicidad ponían en marcha la campaña en redes sociales y hacían la cartelería y el plan de medios para el resto de la campaña (esta vez ya ficticia puesto que se trataba de seleccionar vallas, marquesinas, emisoras de radio, etc.). En esta fase gran parte de los grupos estaban en contacto con las instituciones a las que habían decidido representar, ya que debían responder en tiempo real a los ciudadanos que opinaban sobre la adopción de mascotas o que estaban interesados en el proceso y demandaban información al respecto.

7. En la fase final, los grupos de ficción entregaron los anuncios y los cortos de ficción a las "agencias publicitarias" que les habían contratado y estos se difundieron en las redes sociales elegidas por cada grupo.

A lo largo de todo el proceso, cada uno de los estudiantes tuvo un rol diferente y, por lo tanto, se hizo responsable de diferentes aspectos que iban desde la producción o el montaje de los vídeos al diseño y ejecución de los carteles, redactar los post para las redes sociales, etc.

Para la evaluación final del trabajo las profesoras distribuyeron la puntuación antes del inicio del ejercicio del modo que se expone a continuación:

En Publicidad la práctica supuso un máximo de 7 puntos sobre los 10 de la materia. Se corresponde con cada una de las siguientes fases y resultados del trabajo:

- Entrega de un briefing y estrategia de campaña: 1 punto.

- Entrega de idea fuerza/slogan sobre el tema tratado en las prácticas: 1 punto. 
- Entrega de un guion publicitario: 1 punto.

- Entrega de un cartel sobre el tema tratado en las prácticas: 1 punto.

- Entrega de la planificación de medios y estrategia de redes sociales del tema tratado en las prácticas: 2 puntos.

- Spot: 1 punto. Se evaluaba la adecuación a la campaña, no el spot en sí.

En Ficción, la nota máxima que los estudiantes podían obtener con la realización del ejercicio era de 2,5 puntos sobre los 10 globales de la asignatura. Esta puntuación se distribuyó del siguiente modo:

- Entrega de un informe del proceso con todos los anexos en el que cada estudiante explicaba su rol, dificultades y soluciones, y resultados de su trabajo. Los estudiantes conocían las características de este informe desde el primer día de curso: 1 punto

- Proceso de desarrollo del ejercicio durante el curso: seguimiento de las fases y etapas de trabajo, entregas puntuales en fecha y modo a través de la plataforma Moodle, profundidad en la realización de cada fase de trabajo en el proceso de desarrollo de una Ficción Audiovisual: 1,2 puntos.

- Resultado: 0,3 puntos.

\subsection{Objetivos}

El principal objetivo de la práctica fue el de que el estudiante de las dos materias adquiriese destrezas y habilidades generales en comunicación y particulares en la elaboración de trabajos de ficción y campañas publicitarias. Dichas destrezas debían adquirirse con la realización de un trabajo capaz de cubrir las necesidades de difusión en materia de adopción animal, y que a su vez sirviese para evaluar ambas asignaturas: Ficción Audiovisual y Publicidad Audiovisual. Como objetivos secundarios se tuvieron en cuenta:

El aprendizaje social. El trabajo sirvió para que el propio estudiante se concienciase de la problemática del abandono y el maltrato animal, así como de la necesaria responsabilidad en el cuidado de las mascotas. Una vez 
concienciado del problema, el estudiante se convertía en promotor de esa misma concienciación en su entorno social más próximo.

El aprendizaje colaborativo, el trabajo en equipo y la resolución de conflictos. Fue fundamental para el desarrollo del trabajo, ya que sacar adelante el proyecto era necesariamente responsabilidad de todos los miembros del equipo.

La formación de destrezas comunicativas. En ambas asignaturas, el alumno debía adquirir los conocimientos suficientes para transmitir y comunicar los mensajes adecuados al público objetivo al que iban dirigidos. A su vez, el contacto real con el público de las redes sociales sirvió para la adquisición de habilidades comunicativas y para la resolución eficaz de conflictos. Esa misma exposición pública hizo también que los grupos de trabajo se esforzasen por conseguir proyectos de mayor calidad y por tener un comportamiento más profesional ante la audiencia y las instituciones a las que representaban.

Estos objetivos se relacionan con las destrezas y objetivos de aprendizaje particulares para cada materia expuestos en las guías docentes de sendas asignaturas y en la memoria de grado del título. Ambos documentos (guías docentes y memoria del título) se tuvieron en cuenta para el diseño de la práctica. En Publicidad este ejercicio se correspondió con los siguientes resultados de aprendizaje:

- Crear mensaxes de carácter publicitario, especialmente no que se refire a produtos audiovisuais. Competencias implicadas: A2, A3, A7, B9, C1

- Coñecer e desenvolver as diferentes técnicas de creatividade. Competencias implicadas: A12, B9, C2, C4.

- Saber elaborar unha mensaxe publicitaria de forma autónoma. Competencias implicadas: A1, A2, A7, B9, C1, C2

En Ficción con:

Relacionar as distintas fases do proceso de elaboración dun produto de Ficción Audiovisual. Competencias implicadas: A1, A2, A7, A8, B6, B9 
Relacionar o rol profesional dos principais implicados na elaboración dunha produción audiovisual de ficción. Competencias implicadas: A7, A8, A12, B6 Al margen de estos objetivos, el trabajo pretendía favorecer en el estudiante el desarrollo de competencias transversales y de conocimiento específico. En primer lugar, el estudiante trabajaba para un "cliente real", Io que en principio hizo que lo percibiese como mucho más útil que cualquier otro tipo de producto.

Esto también dificultó el desarrollo del trabajo, ya que los estudiantes tuvieron que responder a necesidades reales en plazos de tiempo sobre los que tenían poco control, y a aspectos éticos y morales a los que se enfrentaron por primera vez. Eso hace que, en general, el alumno se sienta más responsable de su trabajo, y también más presionado por el resultado, ya que desea satisfacer al "cliente" además de conseguir una buena nota en las asignaturas que está cursando.

Por otro lado, la actividad de ApS hizo que se implicasen en mayor medida en la realidad social de las instituciones que trabajan para el cuidado de las mascotas abandonadas, y aprendieron a reconocer el esfuerzo de voluntariado y social que hacen aquellos que participan de este tipo de actividades, a la vez que mejoraron su visión crítica del mundo.

\section{RESULTADOS}

En la exposición de resultados de este texto, se evita conscientemente tener en cuenta las percepciones de alumnos y profesores en las que se basan buena parte de los estudios en ApS (Lorenzo, M..; Mella, I.; García, J. y Valera, C. 2017), a pesar de que se hicieron cuestionarios al final de las prácticas. El motivo es que no resulta un sistema empírico para un proyecto en el que la percepción por parte de estudiantes no se tuvo en cuenta como objetivo en el diseño. Estos datos de percepción se valorarán en las conclusiones finales.

A pesar de que no todos los grupos de alumnos obtuvieron los mismos resultados, algunos de los trabajos realizados en colaboración con protectoras tuvieron una gran visibilidad y una 
repercusión muy positiva en redes sociales. Los resultados de los proyectos se pusieron a disposición de las asociaciones para que lo siguieran utilizando una vez terminado el curso académico y, para evaluar los resultados del trabajo entre los estudiantes, así como su satisfacción con los logros conseguidos con la práctica, se les realizaron cuestionarios al final del cuatrimestre.

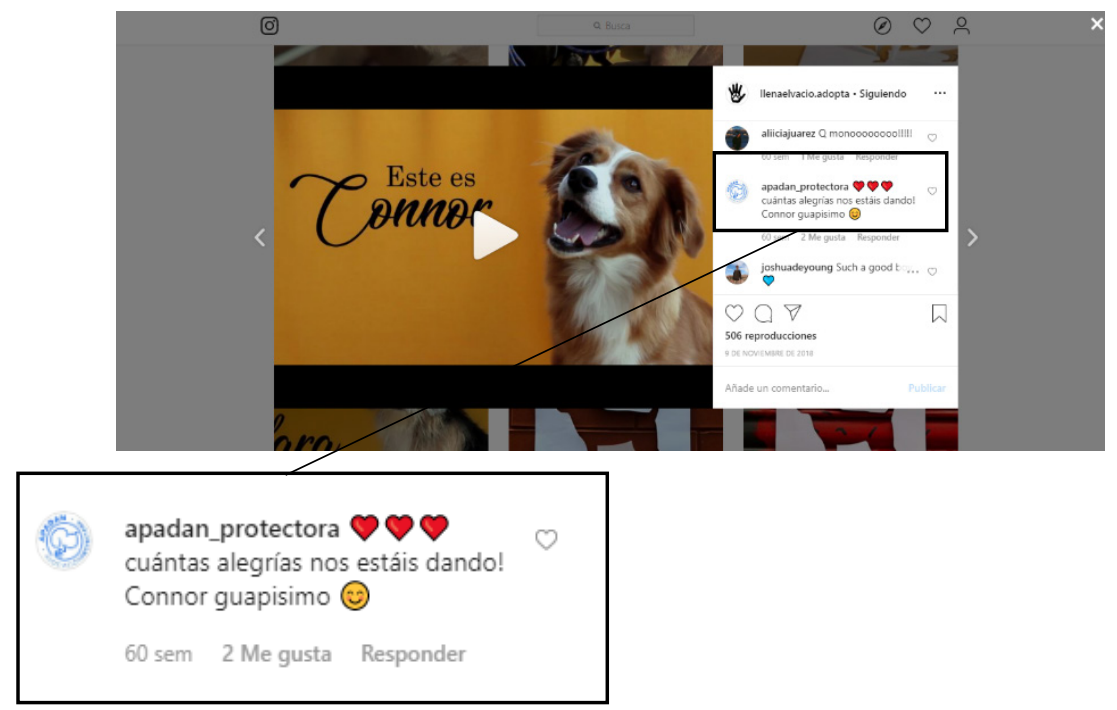

Imagen 1. Ejemplo de la campaña realizada como parte de la experiencia de ApS.

Como resultado del trabajo, los estudiantes elaboraron 8 campañas de publicidad, realizadas en grupos de 6-8 alumnos. En definitiva, llevaron a cabo:

- 8 spots audiovisuales pensados para redes sociales.

- 8 cortos audiovisuales de un minuto, pensados para la participación en festivales y concursos de cortometrajes.

- 8 planes de medios para la realización de la campaña.

- 8 campañas en redes sociales. La elección de las mismas dependía del público objetivo al que iba dirigida cada una de las campañas, por lo que fue variable. Sin embargo, la mayoría de ellos escogieron Instagram, Facebook y Twitter como ventanas de promoción. 
- 8 carteles para utilizar en redes sociales y/o vallas.

- Una memoria final con todo el trabajo realizado.

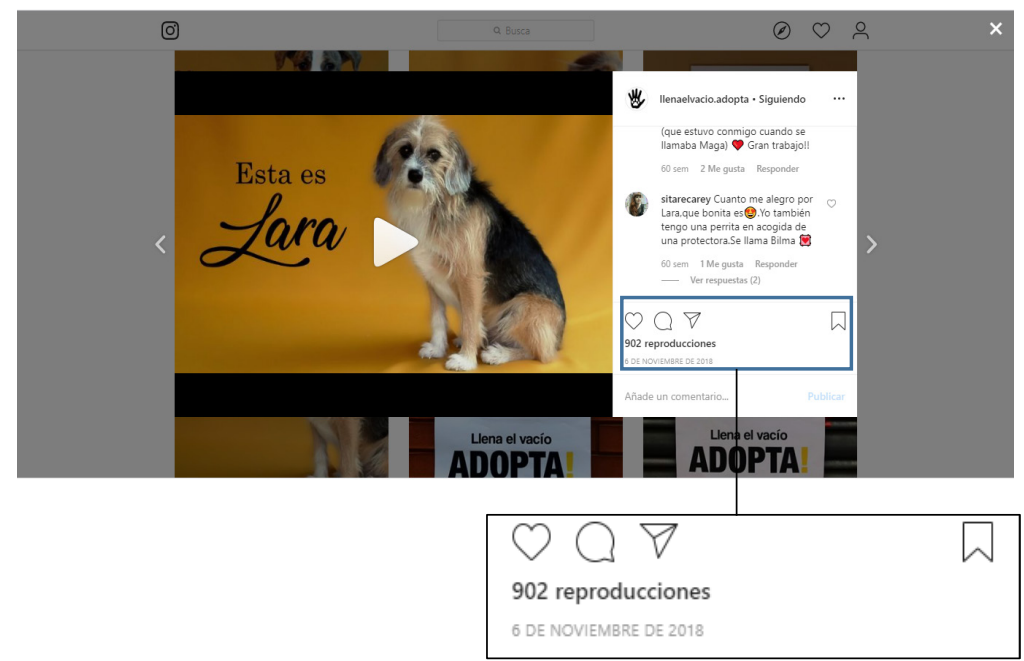

Imagen 2. Ejemplo de la campaña realizada como parte de la experiencia de ApS.

La campaña en las redes sociales se puso en marcha entre la última semana de noviembre y la última de diciembre y los estudiantes tenían la obligación de publicar a diario 0 casi a a diario, por lo que muchos aprovecharon las fechas navideñas para concienciar sobre el regalo de mascotas, muy habitual en esa época del año. A lo largo de ese periodo de tiempo algunas de las campañas realizadas llegaron a alcanzar casi mil seguidores en Instagram y miles de reproducciones a los vídeos promoviendo la adopción, además de decenas de comentarios de usuarios y cientos de likes.

\section{CONCLUSIONES}

Consideramos que el trabajo de innovación docente aquí presentado es interesante y atractivo para los actores involucrados en el proceso (docentes, alumnos e instituciones). Consistió en ofrecer a un organismo un servicio necesario, que buscó la calidad en la medida de lo posible, y en el que el alumno plasmó los conocimientos que fue adquiriendo a lo largo del desarrollo de las dos materias involucradas en el proceso. En este tipo de proyectos, el estudiante mejora 
sus valores cívicos, se involucra con la sociedad y aprende acerca de la responsabilidad de sus acciones (Fernández, Y. \& Martínez, M. J., 2016) al tiempo que mejora habilidades específicas relacionadas con el trabajo en equipo, la comunicación interpersonal y la resolución de conflictos, ya que las contingencias, tanto internas como fuera del aula, han de resolverse en tiempo real. La evaluación que reciben del trabajo no es solo la nota final de ambas materias, sino la percepción del trabajo que el alumno percibe al difundirlo públicamente, lo que hace que se esfuercen más por realizar un trabajo de calidad. En este tipo de proyectos se da también una segunda valoración: la de la institución beneficiaria del proceso, además de la del público general.

Por otro lado, los profesores responsables de las asignaturas implicadas en el proceso se sienten más involucrados en el proyecto al entender que su aportación es también útil a una institución o comunidad (Puig, J.; Batlle, R.; Bosch, C. \& Palos, J., 2007). Las actividades se realizan en un contexto ajeno al aula, y por lo tanto se mejora el contacto con el entorno real del estudiante $y$, a pesar de que en ocasiones supone un trabajo extra, el tipo de proyecto suele resultar mucho más activo y motivador. Sin embargo, del estudio sobre el proceso de implementación de esta práctica de innovación docente se deduce que la respuesta no fue en general tan buena como era deseable, en particular para los estudiantes. En las encuestas de evaluación, los alumnos se quejaron de las exigencias del trabajo, al que dedicaron mucho tiempo y esfuerzo que creían no ver recompensado en la interacción con el público. En ese sentido, la satisfacción del estudiante varió mucho según los resultados obtenidos, en particular en las redes sociales. El trabajo en equipo también les resultó muy complejo, al tratarse de grupos más grandes de lo habitual (8-10 personas), y les costó adaptarse a sus roles en las dos materias, ya que estos iban rotando en función de los días de la semana, lo que les producía una cierta confusión.

Finalmente, consideramos que el trabajo realizado cumplió con los objetivos de una práctica de Aprendizaje-Servicio, pero consideramos que debería mejorarse como práctica conjunta en lo que al aprendizaje basado en proyecto y al trabajo de roles de equipo se refiere. La mezcla 
de los códigos de ficción y publicitario restringió el trabajo en muchos aspectos, como la duración de los vídeos o las fechas de entrega. A pesar de que las docentes consideraron estos aspectos como positivos para la práctica, los estudiantes se quejaron de ello. También obligó a que los alumnos trabajasen en base a un eslogan, lo que les limitó creativamente en algunos aspectos. A pesar de ello, el proyecto sirvió para que apreciasen mejor la problemática relacionada con el abandono y la adopción de las mascotas, aprendieron los aspectos éticos y legales al respecto y mejoraron sus habilidades y destrezas comunicativas de cara a las dos materias. Además, varias de las instituciones que participaron del proceso agradecieron a los estudiantes su participación y su trabajo, tanto públicamente a través de las redes sociales como a nivel particular, lo que sirvió para motivarles.

Por otro lado, en relación a los resultados de aprendizaje planteados en las guías docentes de sendas asignaturas y en la memoria del título, la práctica permitió una aproximación a los mismos basada en una experiencia real. En el caso de ficción tan solo uno de los ocho grupos que realizaron la práctica obtuvo una calificación de insuficiente en la obtención de resultados de aprendizaje del ejercicio en la primera convocatoria. Es cierto que, en contrapartida, solo un grupo obtuvo la calificación máxima en el ejercicio. La mayoría de los grupos, por tanto, obtuvieron una calificación del ejercicio entre aprobado y notable, lo cual se considera un resultado medio adecuado. En la materia de Publicidad, en cambio, ningún grupo obtuvo la máxima cualificación y solo la mitad de ellos consiguieron aprobar la práctica.

\section{REFERENCIAS}

Fernández, Y. \& Martínez, M. J. (2016). Cuando sentimiento y acción confluyen en la práctica educativa. Alianzas entre EpD y ApS para una ciudadanía global. RIDAS, Revista Iberoamericana de Aprendizaje y Servicio, 2, 111-138. DOI10.1344/RIDAS2016.2.6

Francisco, A. \& Moliner, L. (2010). El Aprendizaje Servicio en la Universidad: una estrategia en la formación de ciudadanía crítica. REIFOP, 13 (4). (Enlace web: http://www.aufop.com - Consultada en 12.03.2016) 
Lorenzo, M.; Mella, I.; García, J. y Valera, C. (2017). Investigar para institucionalizar el aprendizaje servicio en la universidad española. RIDAS, Revista Iberoamericana de Aprendizaje y Servicio, 3, 118- 130. D0I10.1344/RIDAS2017.3.9.

Marti, J. A.; Heydrich, M.; Rojas, M.; Hernández, A. (2010) Aprendizaje basado en proyectos: una experiencia de innovación docente. Revista Universidad EAFIT, 46(158), pp. 11-21. Recuperado de http://publicaciones.eafit.edu.co/index.php/revista-universidadeafit/article/view/743

Puig, J., Batlle, R., Bosch, C. \& Palos, J. (2007) ¿Qué es el aprendizaje servicio? Aprendizaje servicio. Educar para la ciudadanía (9-28). Barcelona: Octaedro.

Puig, J., Batlle, R., Bosch, C., de la Cerda, M., Climent, T., Gijón, M., Graell, M., Martín, X., Muñoz, A., Palos, J., Rubio, L.\& Trilla, J.(2009). Aprendizaje Servicio (ApS) Educación y compromiso cívico. Barcelona: Graó.

Sandrea, L. \& Reyes, L. (2010) Proyectos de aprendizaje de servicio-comunitario y su influencia en las conductas prosociales de estudiantes universitarios. Revista de Pedagogía, 31(89), 379-401.

Villa, A \& Villa, 0. (2007). El aprendizaje basado en competencias y el desarrollo de la dimensión social en las universidades. Educar, nº 40, pp. 15-48. 